\title{
Considerações acerca dos aspectos emocionais na vida do paciente oncológico ostomizado
}

\author{
Considerations about the emotional aspects in the living of the ostomized oncological \\ patient \\ Consideraciones sobre los aspectos emocionales en la vida del paciente oncológico \\ ostomizado
}

Silvana Bastos Cogo $^{1}$, Daiane Vargas ${ }^{1}$, Ariele Priebe Reisdorfer ${ }^{1}$, Aline Gomes Ilha ${ }^{1 *}$, Luiza Carolina Santos Malheiros ${ }^{1}$, Elisabeta Albertina Nietsche ${ }^{1}$, Marcio Rossato Badke ${ }^{1}$, Graciela Dutra Sehnem¹.

\section{RESUMO}

Objetivo: Analisar as repercussões emocionais causadas pelo uso de ostomias em pacientes em tratamento oncológico. Métodos: Trata-se de um estudo transversal com abordagem quantitativa. Amostra composta por 30 participantes e coleta de dados realizada de julho a setembro de 2017, nos setores de quimioterapia, radioterapia e clínica médica de um hospital universitário. A coleta de dados foi realizada por meio de um formulário impresso com perguntas abertas e fechadas aplicado aos pacientes no momento de seu tratamento nos respectivos setores do hospital. Resultados: Os resultados evidenciaram a importância do apoio emocional de pacientes e cuidadores, desde o diagnóstico até o período de adaptação a condição de portador de ostomia, já que que a maior parte dos pacientes possuía alterações emocionais e quase a totalidade deles possuía ao menos um sentimento que interferia na rotina diária. Conclusão: Conclui-se que o suporte psicológico deve ser realizado com o paciente e seus familiares para auxiliá-los no enfrentamento às dificuldades vivenciadas durante o tratamento.

Palavras-chave: Emoções, Ostomia, Neoplasias.

\section{ABSTRACT}

Objective: To analyze the emotional repercussions caused by the use of ostomies in patients undergoing cancer treatment. Methods: This is a cross-sectional study with a quantitative approach. Sample composed of 30 participants and data collection carried out from July to September 2017, in the sectors of chemotherapy, radiotherapy and medical clinic of a university hospital. Data collection was performed using a printed form with open and closed questions applied to patients at the time of their treatment in the respective sectors of the hospital. Results: The results showed the importance of emotional support from patients and caregivers, from diagnosis to the adaptation period to the condition of having an ostomy, since most patients had emotional changes and almost all of them had at least one feeling that interfered in the daily routine. Conclusion: It is concluded that psychological support must be performed with the patient and his family to help them cope with the difficulties experienced during treatment.

Keywords: Emotions, Ostomy, Neoplasms.

\section{RESUMEN}

Objetivo: Analizar las repercusiones emocionales que provoca el uso de ostomías en pacientes sometidos a tratamiento oncológico. Métodos: Se trata de un estudio transversal con enfoque cuantitativo. Muestra compuesta por 30 participantes y recogida de datos realizada de julio a septiembre de 2017, en los sectores de quimioterapia, radioterapia y clínica médica de un hospital universitario. La recolección de datos se realizó mediante un formulario impreso con preguntas abiertas y cerradas aplicadas a los pacientes al momento de su tratamiento en los respectivos sectores del hospital. Resultados: Los resultados evidenciaron la

${ }^{1}$ Universidade Federal de Santa Maria (UFSM), Santa Maria - RS. *E-mail: aline.gomes1996@hotmail.com 
importancia del apoyo emocional de los pacientes y cuidadores, desde el diagnóstico hasta el período de adaptación a la condición de tener una ostomía, ya que la mayoría de los pacientes tenían cambios emocionales y casi todos tenían al menos uno. sentimiento que interfería en la rutina diaria. Conclusión: Se concluye que se debe realizar un acompañamiento psicológico con el paciente y su familia para ayudarlos a afrontar las dificultades experimentadas durante el tratamiento.

Palabras clave: Emociones, Ostomía, Neoplasias.

\section{INTRODUÇÃO}

O câncer é designado como uma doença crônico-degenerativa decorrente de modificações no código genético. Conforme estimativas para o triênio 2020-2022, com exceção do câncer de pele não melanoma, os tipos de câncer mais frequentes para esse período serão os cânceres de próstata (65.840 casos novos a cada ano) em homens e mama ( 66.280 casos novos a cada ano) em mulheres. Além dos citados, completarão a lista dos dez tipos de câncer mais incidentes: cólon e reto (intestino - 40.990), pulmão (30.200), estômago (21.230), colo do útero (16.590), cavidade oral (15.190), esôfago (11.390) sistema nervoso central (11.090) e leucemias (10.810) (INCA, 2019).

A incidência de câncer cresceu significativamente no mundo. $O$ câncer de intestino ocupa, a cada dia, mais espaço, sendo atualmente o terceiro mais incidente no mundo (1,8 milhão). No Brasil, conforme apresentado anteriormente, estima-se que o câncer de colo e reto também continuará assumindo a terceira posição na escala das neoplasias mais incidentes (INCA, 2019).

Apesar de todos os progressos científicos e tecnológicos, e da evolução dos meios de diagnóstico e de terapias, o câncer continua a ameaçar a vida daqueles que atinge, afetando seu bem-estar físico e psicológico, bem como a sua vida familiar, social e profissional (OLIVEIRA IMS, 2004). Para além da complexidade da sua origem, o câncer está associado a elevados custos sociais e econômicos, originando um conjunto de reações emocionais ao paciente afetado pela doença, com repercussões imediatas na família e na sociedade.

A redução de mortalidade pelo câncer está associada a períodos mais longos de morbidade da população, gerando assim aumento na expectativa de vida e, consequentemente, a necessidade de utilização de aparatos tecnológicos, como uso de ostomias (CAMARGOS MCS e GONZAGA MR, 2015).

Nessa perspectiva, a ostomização trata-se de um procedimento cirúrgico, capaz de desencadear mudanças importantes no estilo de vida, aspecto físico, psíquico, social e espiritual da pessoa submetida à cirurgia, alterando sua percepção na autoimagem e autoestima (FREIRE DA, et al., 2017; KIMURA CA, et al., 2017).

As ostomias mais realizadas, oriundas em decorrência de neoplasias malignas, são as intestinais, traqueais e urinárias. A colostomia é um dos tipos de ostomia intestinal mais frequentemente utilizada e caracteriza-se pela exteriorização do colón através da parede abdominal, com o objetivo de realizar a eliminação fecal (SELAU CM, et al., 2019). A ostomização pode ser transitória ou permanente (FERREIRA EC, 2017). Devido à característica do estoma digestivo, o paciente não possui o controle da eliminação de fezes e gases, o que gera grande impacto emocional desencadeando alterações na sua percepção corporal e autoestima (FREIRE DA, et al., 2017).

Destaca-se que, atualmente, a sociedade estabelece padrões de beleza corporal, e através da alteração física provocada pela ostomia, essa pessoa não se enquadraria nos padrões sociais instituídos, o que pode Ihe desencadear a sensação de rejeição (MOREIRA WC, et al, 2017). Assim, o processo de ostomização, provoca o surgimento de sentimentos ambivalentes e conflituosos em quem o vivencia, podendo inibir a adaptação e a aceitação da atual condição de vida.

Para Santos VLCG e Cesaretti IUR (2005), é por intermédio da imagem corporal que o indivíduo mantém o equilíbrio interno, ao mesmo tempo em que interage com o ambiente, uma vez que é ela que lhe proporciona o senso de identidade e influência na habilidade e execução das atividades da vida diária (AVDs). 
O estado emocional do paciente anteriormente e imediatamente após a cirurgia pode apresentar sintomas de ansiedade e depressão, os quais podem contribuir de forma negativa no estabelecimento de novas relações sociais, além de potencializar o medo, a dor e o sofrimento (CEREZETTI CRN, 2012). Cabe ressaltar, então, a relevância de suporte psicológico imediato, ou seja, logo após a cirurgia para realização de ostomia ter sido indicada como medida imprescindível para continuidade do tratamento (CEREZETTI CRN, 2012).

A literatura, no que tange a compreensão da condição emocional de pacientes ostomizados, é restrita. No entanto, refere-se a um tema que merece estudos destinados, especialmente, a compreender as necessidades das pessoas envolvidas, e à melhoria e ao desenvolvimento de técnicas para auxiliar no manejo e enfrentamento da situação (CEREZETTI CRN, 2012).

Diante da complexidade do tratamento e da reabilitação do ostomizado, este estudo tem como objetivo analisar as repercussões emocionais causadas pelo uso de ostomias em pacientes em tratamento oncológico.

\section{MÉTODOS}

Trata-se de um estudo quantitativo, transversal, realizado no período compreendido entre julho a setembro de 2017, em um hospital universitário localizado no Rio Grande do Sul (RS), no Brasil. Os setores que contemplaram o estudo foram: quimioterapia, radioterapia e unidade de internação (clínica médica I).

A pesquisa foi aprovada pelo Comitê de Ética em Pesquisa da Universidade Federal de Santa Maria, sob o parecer n. 2.121.610.

O local da pesquisa conta com 403 leitos divididos em 40 especialidades; desses, 47 são destinados aos pacientes oncológicos. Além disso, oferecem tratamento antineoplásico a, em média, 360 pacientes/mês no ambulatório de quimioterapia e 40 pacientes/mês no ambulatório de radioterapia. Esta instituição é referência no atendimento a pacientes oncológicos, sendo caracterizado como Unidade de Alta Complexidade em Oncologia (Unacon) nos Serviços de Radioterapia, Hematologia e Oncologia Pediátrica.

A amostra se deu por conveniência não probabilística, e foi composta conforme os critérios de inclusão, por indivíduos de ambos os sexos com idade igual ou superior a 18 anos, com o diagnóstico confirmado de neoplasia, em tratamento quimioterápico e/ou associado à radioterapia, em uso de qualquer tipo de ostomia. Além disso, eles encontravam-se em condições físicas e cognitivas para responderem ao instrumento necessário à coleta de dados. Assim, os indivíduos em condições físicas e cognitivas limitadas para responderem ao instrumento, foram excluídos da pesquisa.

A coleta de dados foi realizada por meio de um formulário impresso com perguntas abertas e fechadas aplicado aos pacientes no momento de seu tratamento nos respectivos setores do hospital, sendo estabelecido o contato pessoal com eles, convidando e informando-os sobre os objetivos e a relevância da pesquisa. Após, assinaram o termo de consentimento livre e esclarecido (TCLE) concordando com o uso das informações coletadas para fins acadêmicos.

Foram coletados dados sociodemográficos (idade, gênero, escolaridade, estado civil e renda), sobre o estilo de vida, sobre os aspectos emocionais e as alterações no humor e sobre os padrões de vida, por meio de um formulário específico elaborado pelas pesquisadoras.

Os dados clínicos (diagnóstico clínico, comorbidades associadas e tipo de ostomia) foram coletados do prontuário físico e eletrônico do paciente. Os dados coletados foram digitados em uma planilha do Software Microsoft Exce周, versão 2016, com dupla digitação e avaliação de compatibilidade dos dados. Após essa etapa, foram realizadas análises de frequência absoluta e relativa no Software SPSS 21.0.

\section{RESULTADOS}

A amostra do estudo foi composta por 30 pessoas, predominantemente de homens (70\%), casados (50\%), com ensino fundamental incompleto $(73,3 \%)$ e com renda inferior a três salários mínimos $(86,7 \%)$. As características sociodemográficas da amostra do estudo estão demonstradas na Tabela 1. 
Tabela 1 - Características sociodemográficas da amostra do estudo, $n=30$.

\begin{tabular}{|c|c|c|}
\hline Variáveis & $\mathbf{N}$ & $\%$ \\
\hline \multicolumn{3}{|l|}{ Gênero } \\
\hline Masculino & 21 & 70,0 \\
\hline Feminino & 9 & 30,0 \\
\hline \multicolumn{3}{|l|}{ Faixa etária } \\
\hline Adulto & 15 & 50,0 \\
\hline Idoso (>60 anos) & 15 & 50,0 \\
\hline \multicolumn{3}{|l|}{ Estado Civil } \\
\hline Solteiro & 7 & 23,3 \\
\hline Casado & 15 & 50,0 \\
\hline Divorciado & 2 & 6,7 \\
\hline Viúvo & 4 & 13,3 \\
\hline União estável & 1 & 3,3 \\
\hline \multicolumn{3}{|l|}{ Escolaridade } \\
\hline Analfabeto & 1 & 3,3 \\
\hline Ensino fundamental completo & 2 & 6,7 \\
\hline Ensino fundamental incompleto & 22 & 73,3 \\
\hline Ensino médio completo & 4 & 13,3 \\
\hline Ensino médio incompleto & 1 & 3,3 \\
\hline \multicolumn{3}{|l|}{ Renda } \\
\hline Nenhuma renda & 1 & 3.3 \\
\hline Até um Salário & 11 & 36.7 \\
\hline De um a três salários & 14 & 46.7 \\
\hline De 3 a 6 salários & 4 & 13,3 \\
\hline \multicolumn{3}{|l|}{ Acompanhante } \\
\hline Com acompanhante & 27 & 90,0 \\
\hline Sem acompanhante & 3 & 10.0 \\
\hline
\end{tabular}

Fonte: Cogo SB, et al., 2020.

A principal doença que gerou a confecção do estoma foi a neoplasia de cólon, seguido de neoplasia de cabeça e pescoço. O tipo de ostomia prevalente foi a colostomia e, $40 \%$ dos pacientes estavam com a ostomia por período superior a 6 meses. Esses dados são observados na Tabela 2.

Tabela 2 - Características clínicas da amostra do estudo, $\mathrm{n}=30$.

\begin{tabular}{lll}
\hline Variáveis & N & $\%$ \\
\hline Diagnóstico Clínico & & \\
\hline Neoplasia Colorretal & 16 & 53,0 \\
Neoplasia Cabeça e pescoço & 9 & 30,0 \\
Neoplasia Estômago & 3 & 10,0 \\
Neoplasia Linfoma não Hodking & 1 & 3,0 \\
Neoplasia Bexiga & 1 & 3,0 \\
\hline Tipos de Ostomia & & \\
\hline Traqueostomia & 8 & 26,7 \\
Gastrostomia & 82 & 6,7 \\
Jejunostomi & 3 & 10,0 \\
Colostomia & 16 & 53,3 \\
lleostomia & 1 & 3,3 \\
Urostomia & 1 & 3,3 \\
\hline Período & & \\
\hline < 1 mês & 2 & 6,7 \\
De 1 - 3 meses & 9 & 30,0 \\
De 3 - 6 meses & 7 & 23,3 \\
Mais de 6 meses & 12 & 40,0 \\
\hline
\end{tabular}

Fonte: Cogo SB, et al., 2020. 
Quanto às alterações no cotidiano, 17 afirmaram ter ocorrido mudanças no seu modo de vida $(56,7 \%), 14$ alegaram ter impactado em suas AVDs (46,7\%) e 24 participantes afirmaram que algum sentimento interfere no desempenho de suas atividades diárias após a cirurgia (80,0\%), conforme apresentado na Tabela 3.

Tabela 3 - Caracterização de sentimentos e percepções após colocação da ostomia, n=30.

\begin{tabular}{lcc}
\hline Variáveis & $\%$ & N \\
\hline Alterações emocionais & 56,7 & 17 \\
Alterações AVDs & 46,7 & 14 \\
Alterações no lazer & 40,0 & 12 \\
\hline Conviver com a ostomia & & 2 \\
\hline Ótimo & 6,6 & 8 \\
Bom & 26,7 & 12 \\
Regular & 40,0 & 8 \\
Ruim & 26,7 & 24 \\
Algum sentimento interfere no dia-a-dia & 80,0 & 7 \\
Apresenta sentimento de alegria & 23,3 & 5 \\
Apresenta sentimento de gratidão & 16,7 & 5 \\
Apresenta sentimento de satisfação & 16,7 & 7 \\
Apresenta sentimento de insegurança & 23,3 & 5 \\
Apresenta sentimento de medo & 16,7 & 5 \\
Apresenta sentimento de vergonha & 16,7 & 9 \\
Apresenta sentimento de revolta & 30,0 & 9 \\
Apresenta sentimento de raiva & 26,7 & 3 \\
Apresenta sentimento de angústia & 30,0 & 1 \\
Apresenta sentimento de nojo & 10,0 & 9 \\
Apresenta sentimento de mágoa & 3,3 & \\
Apresenta sentimento de vida sexual atualmente - ativa & 30,0 & \\
\hline
\end{tabular}

Fonte: Cogo SB, et al., 2020.

\section{DISCUSSÃO}

Em relação às características sociodemográficas dos participantes, foi possível perceber que se assemelham a outros estudos sobre pessoas com ostomia, as quais são predominantemente do sexo masculino, idosos, com baixo nível de escolaridade, e o estado civil é variável (FERREIRA EC, 2017).

O tipo de ostomia prevalente foi a colostomia devido à neoplasia de cólon, o que confirma os dados da literatura afirmando que as ostomias intestinais são as mais comuns de serem encontradas (SELAU CM, et al., 2019). No que se refere ao tempo do estoma, somente $40 \%$ dos participantes tinham sido submetidos à sua confecção há mais de seis meses. Esse dado diverge de outro estudo realizado com pacientes que possuíam colostomia definitiva por câncer colorretal, os quais somente $5 \%$ possuíam o estoma há menos de um ano (MACIEL DBV, et al., 2019).

O diagnóstico e o tratamento do câncer afetam a vida do paciente, causando efeitos colaterais indesejados e mudanças consideráveis nos hábitos diários. Ocorre, por vezes, que o paciente portador dessa doença se torna dependente de cuidados de terceiros, sofre mudanças que geram insatisfação com sua imagem corporal, isola-se socialmente e se afasta de atividades de lazer, o que pode desencadear sintomas de depressão e outros distúrbios psicológicos (SANTANA JJRA, et al., 2008). Diante disso, foi possível verificar, neste estudo, que a maior parte dos pacientes possuía alterações emocionais e que quase a totalidade deles possuía ao menos um sentimento que interferia no dia a dia, como insegurança, angústia e revolta.

Esses aspectos encontrados corroboram com estudo realizado em um hospital no Sul do país, com o objetivo de conhecer o processo de adaptação para a vivência com estomas intestinais, no qual foi possível observar que os pacientes apresentavam sentimentos negativos com relação ao corpo, a citar: a insegurança, o constrangimento e até mesmo, a discriminação (REISDORFER N, et al., 2019). Essas alterações demonstram que o processo de adoecimento e a realização de estomas implicam na descontinuidade da rotina exercida pelo sujeito diariamente, alterando valores, prioridades e projetos de vida. Neste sentido, o 
tornar-se ostomizado atinge também a família dessa pessoa, a qual estabelece redes de apoio e desenvolvimento para o seu cuidado (SIMON BS, et al., 2015).

Nesse contexto, quando alguém recebe uma notícia impactante, como a necessidade de confecção do estoma, entra em um processo de perda onde vai desenvolver as denominadas cinco fases do luto: a negação, a raiva, a barganha, a depressão e a aceitação. Essas fases não seguem necessariamente esta ordem, e nem todos os pacientes precisam experimentar todas elas (KÜBLER-ROSS E, 2008). Ao compreender as fases desenvolvidas, o sujeito dará novo sentido à vida a partir das próprias perdas. Assim, decidir sobre a própria vida e morte é se apropriar sobre preferências e tomadas de decisão; é se tornar responsável por si mesmo (JARAMILLO IF, 2006).

Em relação à fase da negação, é possível observar esse sentimento em estudo realizado na Espanha com 21 pacientes que receberam a notícia de que deveriam realizar um estoma intestinal. Aqueles no qual a patologia que levaria à confecção do estoma era o câncer, apresentaram reação de rejeição ao procedimento. A realização de um estoma, que ocasiona alterações não somente fisiológicas, mas também psicológicas, pode reforçar a gravidade da patologia, a qual ainda é muito associada à morte, o que explica esse sentimento gerado nos pacientes (HUESO-MONTORO C, et al., 2016).

Portanto, para o paciente oncológico, a dificuldade em lidar com as emoções se torna ainda maior, pois além de enfrentar as dificuldades trazidas pela doença, ainda precisa se apropriar das mudanças em seu corpo e em sua vida provocadas pela presença do estoma, geralmente em curto espaço de tempo.

O processo de enfrentamento da doença associado ao uso de ostomia deve ser acompanhado pela equipe multiprofissional, objetivando a adaptação à condição de portador de ostomia, retorno do paciente às atividades sociais, laborativas e de lazer anteriores à cirurgia. A adaptação à condição de portador de ostomia e da bolsa coletora é um processo relacionado à doença de base, ao grau de incapacidade, aos valores e ao tipo de personalidade individual. Nesse momento, sentimentos negativos como vergonha, repulsa, desesperança e receio de rejeição pelos familiares podem aflorar.

Em relação às AVDs e às atividades de lazer, foram observadas alterações em mais de $40 \%$ da população estudada. Esse dado vai ao encontro de estudo realizado com 17 pessoas com estoma, no interior do Rio Grande do Sul, as quais relataram que foi necessário realizar adaptações para realizar as atividades do cotidiano, como tarefas domésticas, atividades de lazer e prática de esportes, após a confecção do estoma intestinal (SELAU CM, et al., 2019). Esse fato pode estar associado à incorporação do estigma social, tendo dificuldades na sua própria aceitação e no seu processo de adaptação (CEREZETTI CRN, 2012).

Reitera-se queos pacientes que foram submetidos à ostomia apresentam alteração de sua perspectiva de vida devido à aparência física e imagem corporal negativa, e podem ainda sofrer mudanças sociais e emocionais, levando a um desequilíbrio emocional, situações essas que podem levar a pessoa ostomizada a um isolamento social (BENÍCIO CDAV, et al., 2016; MOREIRA WC, et al., 2017).

Em relação à atividade sexual, somente $30 \%$ dos entrevistados relataram que a retomaram após a confecção do estoma. Essa situação pode estar relacionada com os sentimentos como perda de autoestima e mudanças no aspecto físico de seu corpo. Porém, o próprio procedimento cirúrgico desencadeia dificuldades ao retorno da atividade sexual, pois pode causar redução da libido e modificações na ereção e ejaculação, nos homens, e estenose ou até mesmo perfuração vaginal, nas mulheres (MOREIRA WC, et al., 2017).

Estudo realizado sobre sexualidade em pacientes ostomizados, em Teresina/PI, demonstrou que 54,3\% deles não exerciam a atividade sexual e 47,4\% relataram diminuição do desejo sexual depois da confecção do estoma. Nesses casos, as alterações psíquicas, como medo de rejeição e sensação de mutilação, provocadas pela modificação na sua imagem corporal, influenciou negativamente em suas atividades sexuais (MOREIRA WC, et al., 2017).

No que se refere a esse aspecto, o papel do psicólogo é fundamental, pois irá auxiliar o paciente na constituição de uma nova imagem de corpo que precisa ser reconhecida, sendo este um processo subjetivo, social e que exige profundas reflexões sobre a convivência com uma ostomia (BARBUTTI RCS, et al., 2008). 
As estratégias de enfrentamento, mediadas pelo psicólogo, podem ser denomiadas como padrões diretos e padrões indiretos. Na primeira, centra-se no problema, na qual o paciente usa habilidades para administrar ou solucionar o problema, são criadas estratégias adptativas, voltadas para a realidade do sujeito. Já na segunda estratégia, centra-se na emoção, ou seja, o paciente utiliza estratégias de enfrentamento que substituam ou regulam o impacto emocional causado pela doença (LORENCETTI A e SIMONETTI JP, 2005).

Destaca-se que, após o diagnóstico de uma condição que provocará a confecção de um estoma, a pessoa pode reagir de diversas formas. Além disso, a adaptação a um acontecimento específico, como no caso do estoma, depende de uma série de fatores que incluem as características pessoais de cada um, seus aspectos emocionais, culturais e de experiências vividas (COELHO AR, et al., 2013). Cada pessoa reage de forma diferenciada aos estímulos de dor e sofrimento e nesse cenário, momentos de solidão, raiva, frustração e depressão também são facilitadores importantes a fim de auxiliar na adaptação à nova imagem.

Nesse sentido, o uso da ostomia torna-se um estressor, que por si só, é um indicador de risco de dificuldades psicológicas e adaptativas. Essa abordagem dificulta a compreensão dos fatores envolvidos na maneira do paciente manejar o estressor, dispersando a aetnção de aspectos que auxiliam para aumentar ou diminuir sua exposição em determinadas situações. Para elaborar programas mais efetivos de intervenção psicológica é necessário identificar os possíveis estressores no contexto de cada paciente, e compreender que há outras variáveis implicadas no processo de enfrentamento do adoecimento (SOUZA J e SEIDL EMF, 2014).

Nessa perspectiva, adquirir conhecimentos acerca do câncer contribui na tomada de decisões do paciente frente ao seu tratamento, informando familiares e amigos, realizando planejamentos e, assim, desenvolvendo maior autonomia. Atrelado a isso, reconstruir as representações acerca da doença e do tratamento pode auxiliar na gestão da ansiedade, já que o problema passa a ser objetivo, oportunizando que os sentimentos relacionados à doença sejam trabalhados, por exemplo, o medo da morte (FONSECA R e CASTRO MM, 2016). Diante do exposto, o psicólogo deve auxiliar o paciente oncológico ostomizado e sua família no momento do diagnóstico, informando sobre a doença, esclarecendo os tratamentos e possíveis efeitos colaterias, ofertando suporte para lidar com eles. Dessa forma, o apoio psicológico ao paciente ostomizado é necessário desde o pré-operatório a fim de auxiliá-lo a aceitar as mudanças na sua condição de vida e aumentar sua autoestima, e dessa forma, evitar complicações e possibilitar avanços no processo de reabilitação (SELAU CM, et al., 2019).

A psicologia trabalha para auxiliar na conscientização do paciente e no seu autoconhecimento. É pela ressignificação de suas crenças, da mudança dos parâmetros com os quais percebia a si e aos outros, ou seja, é pela construção de uma nova narrativa pessoal que realmente se dá a modificação em seu estilo de vida, prevenindo, dessa forma, o acometimento do corpo e adaptação após a cirurgia. No que tange à terapêutica de reabilitação do paciente, deve-se ter como objetivo, principalmente, a retomada deste para as atividades desenvolvidas em seu cotidiano e à autonomia, contribuindo, assim, para que ele utilize seus recursos de enfrentamento e os modifique em vista de um uso mais maduro e condizente com seus objetivos de melhora. Frente a esse processo, aos poucos o paciente percebe que a mudança ocorrida deu - se apenas em sua condição física, e que ele continua a mesma pessoa de sempre, produtiva e capaz de superações e transformações.

Considerando a família como uma das principais fontes de apoio para o paciente oncológico ostomizado, a atuação do psicólogo também deve ser voltada ao suporte a esses familiares, acolhendo suas demandas e orientando-os sobre como apoiar o paciente no decorrer do tratamento. É fundamental que esse apoio seja iniciado desde a comunicação do diagnóstico inicial, a fim de fornecer suporte ao paciente e aos familiares para lidar com as dificuldades vivenciadas durante o tratamento e que se estenda ao período de adaptação e mudanças, como no caso de conviver com uma ostomia. No entanto, compreende-se que ainda não se percebe como essencial o apoio psicológico a esses pacientes, tendo em vista que a maioria não possui acompanhamento no período de internação hospitalar e após a cirurgia. Assim sendo, cabe ressaltar a importância da elaboração destes processos a nível intrapsíquico, a fim de facilitar a compreensão deste processo e, desta forma, conseguir se adaptar e retomar a vida existente anterior à cirurgia, da forma mais natural quanto possível. 


\section{CONCLUSÃO}

A realização de ostomias em pacientes oncológicos leva a alterações emocionais, AVDs, lazer e sentimentos no dia-a-dia. Destaca-se, que nesse contexto, os familiares e cuidadores são as pessoas mais próximas. Dessa maneira, o suporte psicológico deve ser realizado com o paciente e seus familiares para auxiliá-los no enfrentamento às dificuldades vivenciadas durante o tratamento. O presente estudo apresenta limitações devido à amostra restrita. No entanto, os resultados desta pesquisa podem subsidiar profissionais da saúde na assistência aos pacientes ostomizados, bem como suscitar a realização de novas pesquisas sobre o tema.

\section{REFERÊNCIAS}

1. BARBUTTI RCS, et al. Ostomia: Uma difícil adaptação. Revista SBPH, 2008; 11(2):27-39.

2. BENÍ́CIO CDAV, et al. Conviviendo con una estoma húmedo: un estudio acerca de la calidad de vida. Cultura de los Cuidados, 2016; 20(46):165-170.

3. CAMARGOS MCS, GONZAGA MR. Viver mais e melhor? Estimativas de expectativa de vida saudável para a população brasileira. Cad. SaúdePública, 2015; 31(7):1460-1475.

4. CEREZETTI, CRN. Orientações Psicológicas e capacidade reativa de pessoas ostomizadas e seus familiares. $O$ Mundo da Saúde. 2012; 36(2):332-339.

5. COELHO AR, et al. A estomia mudando a vida: enfrentar para viver. Ver. Min. Enferm., 2013; 17(2):258-267.

6. FERREIRA EC, et al. Self-esteem and health-related quality of life in ostomized patients. Rev Bras Enferm., 2017; 70(2):271-278.

7. FONSECA R, CASTRO MM. A importância da atuação do psicólogo junto a pacientes com câncer: uma abordagem psico-oncológica. Psicologia e Saúde em Debate, 2016; e. especial: 54-72.

8. FREIRE DA, et al. Autoimagem e autocuidado na vivência de pacientes estomizados: o olhar da enfermagem. Rev Min Enferm., 2017; 21(e.1019).

9. INSTITUTO NACIONAL DE CÂNCER (Brasil). Estimativas 2020: Incidência de Câncer no Brasil. Rio de Janeiro: INCA, 2019.

10. HUESO-MONTORO C, et al. Experiences and coping with the altered body image in digestive stoma patients. Rev. Latino-Am. Enferm., 2016; 24(e.2840).

11. JARAMILLO IF. Morrer Bem. São Paulo: Editora Planeta, 2006.

12. KIMURA CA, et al. Quality of life analysis in ostomized colorectal cancer patients. Journal of Coloproctology, 2013; 33(4):216-221.

13. KÜBLER-ROSS E. Sobre a Morte e o Morrer: o que os doentes terminais têm para ensinar a médicos, enfermeiras, religiosos e aos próprios parentes. 9. ed. São Paulo: Martins Fontes, 2008.

14. LORENCETTI A, SIMONETTI JP. As estratégias de enfrentamento de pacientes durante o tratamento de radioterapia. Revista Latino-americana de Enfermagem, 2005; 13(6):944-950.

15. MACIEL DBV et al. Perfil sociodemográfico de pacientes com estomia definitiva por câncer colorretal: interferência na qualidade de vida. Nursing, Säo Paulo, 2019; 22(258):3339-3344.

16. MOREIRA WC, et al. Sexualidade de pacientes com estomias intestinais de eliminação. J. res.: fundam. Care, 2017; 9(2):495-502.

17. OLIVEIRA IMS. Vivências da mulher mastectomizada: Abordagem fenomenológica da relação com o corpo. Escola Superior de Enfermagem Imaculada Conceição. Universidade do Porto, Porto, 2004.

18. REISDORFER N, et al. Processo de transição para vivência com estomias intestinais de eliminação: repercussões na imagem corporal. Estima Braz. J. EnterostomalTher., 2019; 16(e.1219).

19. SANTANA JJRA, et al. Pacientes com câncer: enfrentamento, rede social e apoio social. Paidéia, 2008; 18(40):371384.

20. SANTOS VLCG, CESARETTI IUR. Assistência em Estomaterapia, Cuidando do Ostomizado. [S. I.]: EditoraAtheneu, 2005.

21. SELAU CM, et al. Percepção dos pacientes com estomia intestinal em relação às mudanças nutricionais e estilo de vida. TextoContextoEnferm., 2019; 28(e.20180156).

22. SIMON BS, et al. "Sempre ajudando em uma coisa ou outra: rede social da família da pessoa com estomia. Rev.Eletr. Enf., 2015; 17(2):370-378.

23. SOUZA J, SEIDL EMF. Distress e enfrentamento: da teoria à prática em psico-oncologia. Brasília Med., 2014; 50(3):242-252. 